\title{
THE SCALE OF THE SPREAD OF COVID-19 IN GEORGIA AND EFFECTIVENESS OF PREVENTIVE MEASURES IMPLIMENTED BY THE GOVERNMENT - WHAT DO MODELS SAY?
}

\author{
IURI ANANIASHVILI \\ Doctor of Economic Sciences, Professor, \\ Academician of Georgia Economic Sciences Academy \\ Ivane Javakhishvili Tbilisi State University, Georgia \\ luri.ananiashvili@tsu.ge
}

\section{LEVAN GAPRINDASHVILI}

Master of Economics,

Ivane Javakhishvili Tbilisi State University

levani.gafrindashvili@gmail.com

Abstract. In this article we present forecasts of the spread of COVID-19 virus, obtained by econometric and machine learning methods. Furthermore, by employing modelling method, we estimate effectiveness of preventive measures implemented by the government. Each of the models discussed in this article is modelling different characteristics of the COVID-19 epidemic's trajectory: peak and end date, number of daily infections over different forecasting horizons, total number of infection cases. All these provide quite clear picture to the interested reader of the future threats posed by COVID-19.

In terms of existing models and data, our research indicates that phenomenological models do well in forecasting the trend, duration and total infections of the COVID- 19 epidemic, but make serious mistakes in forecasting the number of daily infections. Machine learning models, deliver more accurate short -term forecast of daily infections, but due to data limitations, they struggle to make long-term forecasts. Compartmental models are the best choice for modelling the measures implemented by the government for preventing the spread of COVID-19 and determining optimal level of restrictions. These models show that until achieving herd immunity (i.e. without any epidemiological or government implemented measures), approximate number of people infected with COVID-19 would be 3 million, but due to preventive measures, expected total number of infections has reduced to several thousand (1555-3189) people. This unequivocally indicates the effectiveness of the preventive measures.

KEYWORDS: CORONAVIRUS, FORECASTING, COMPARTMENTAL MODELS, RICHARDS MODEL, POLYNOMIAL MODEL, NEURAL NETWORK MODEL, BASIC REPRODUCTION NUMBER.

For citation: Ananiashvili, I., \& Gaprindashvili, L., (2020). The Scale of the Spread of COVID -19 in Georgia and Effectiveness of Preventive Measures Implemented by the Government - What Do Models Say? Globalization and Business, $10.49-57$. https://doi.org/10.35945/gb.2020.10.005 


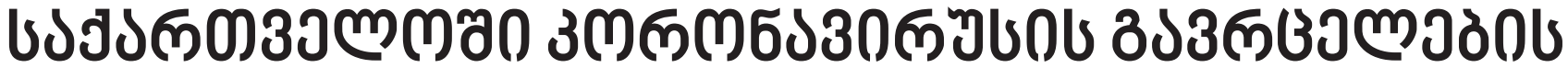

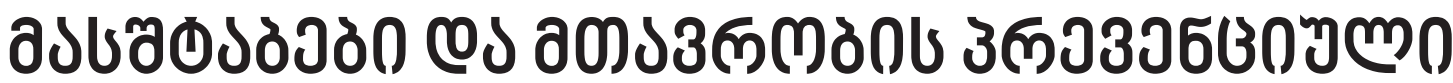

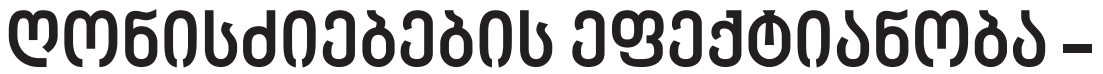

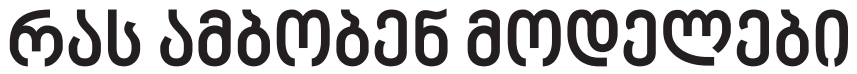

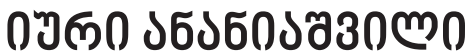

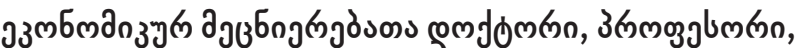

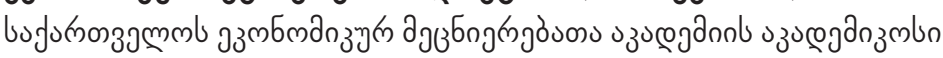

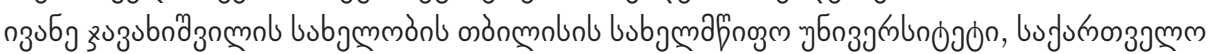 \\ luri.ananiashvili@tsu.ge
}

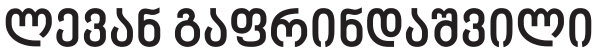

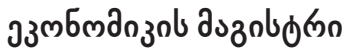

levani.gafrindashvili@gmail.com

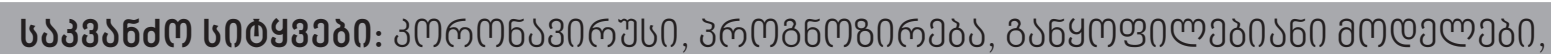

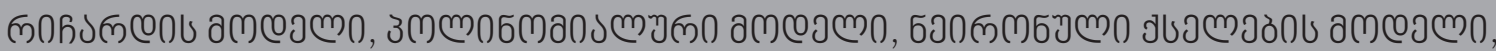

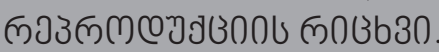

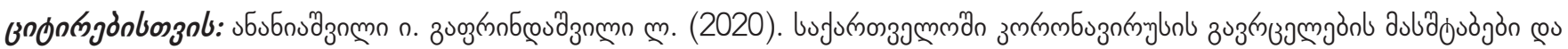

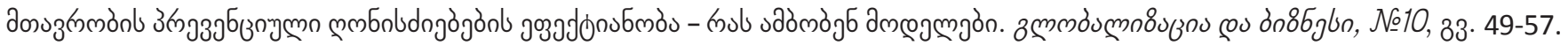
https://doi.org/10.35945/gb.2020.10.005

\section{əอง১3১ल00}

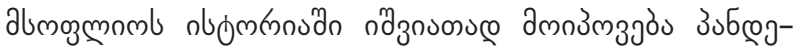

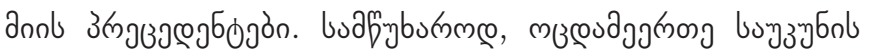

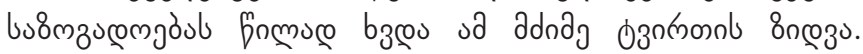

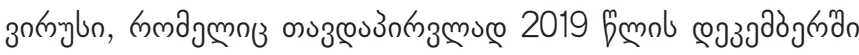

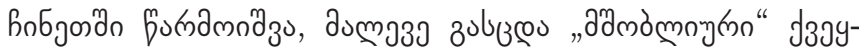

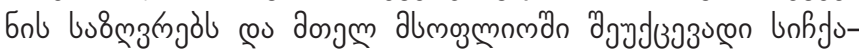

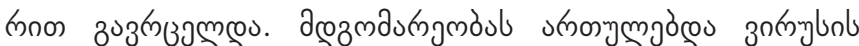

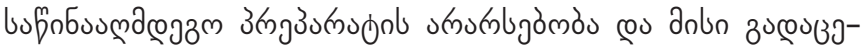

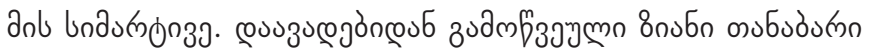

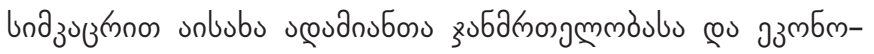

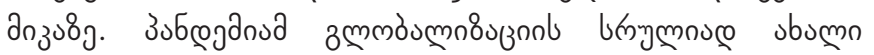

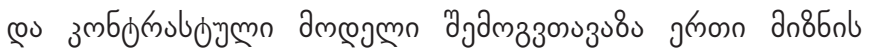

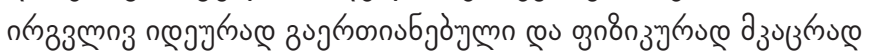

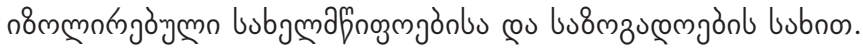

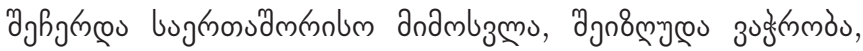

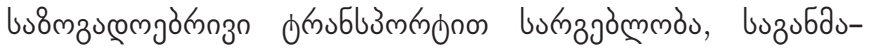

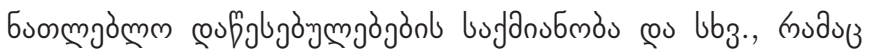

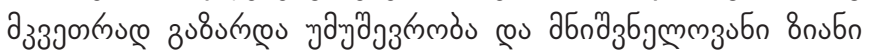

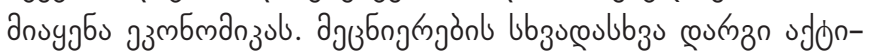

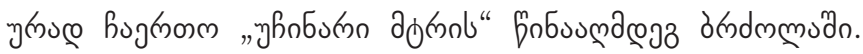

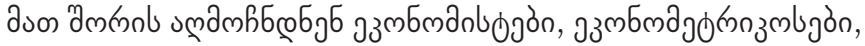

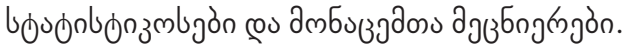

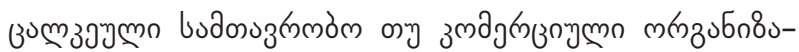

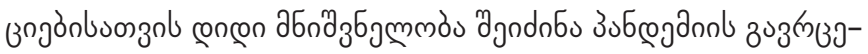

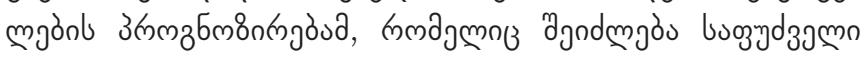

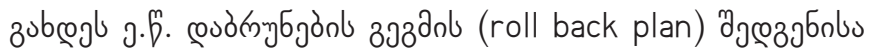

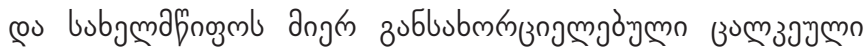

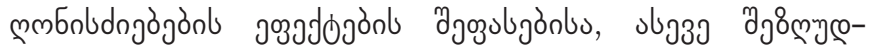

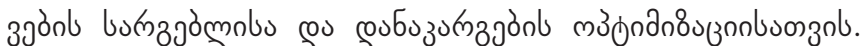

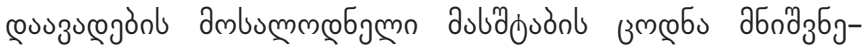

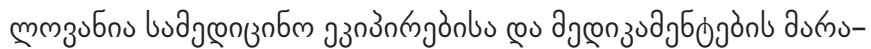

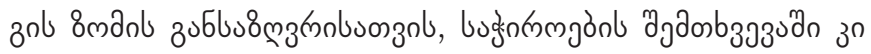

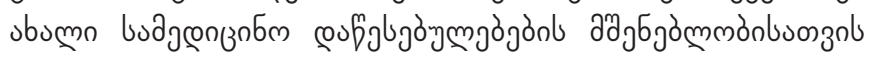

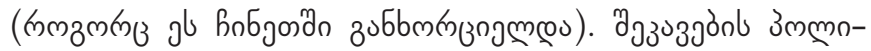

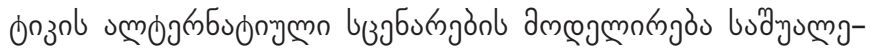

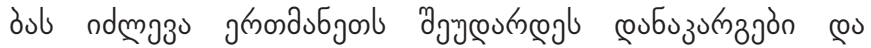

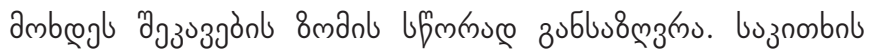

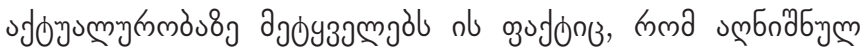

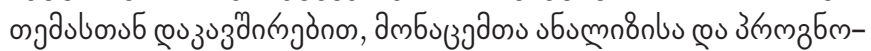

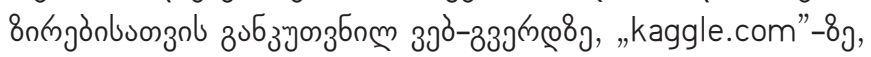

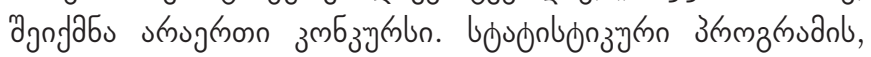

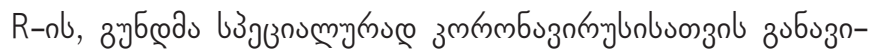




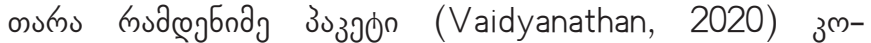

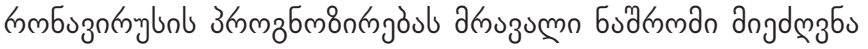

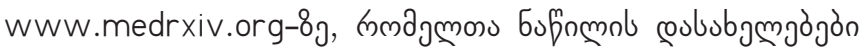

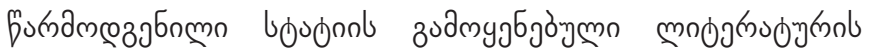

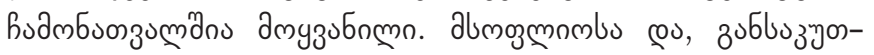

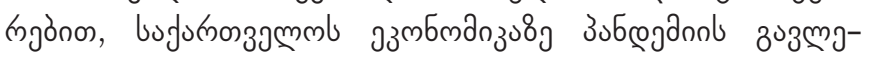

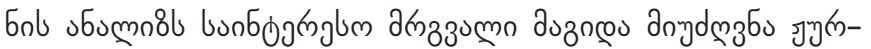

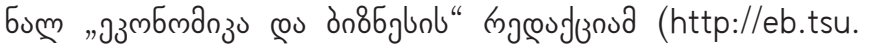

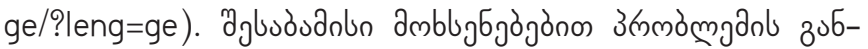

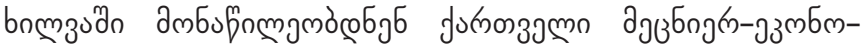

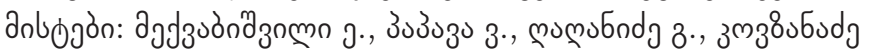

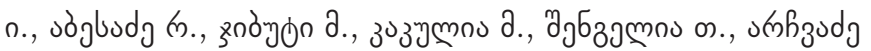

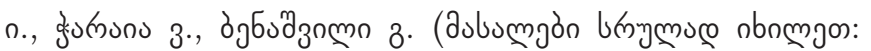

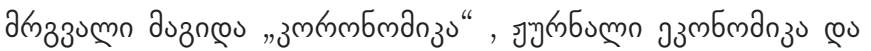
ठ̀n85glo, 2020, №2).

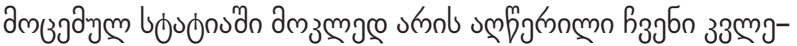

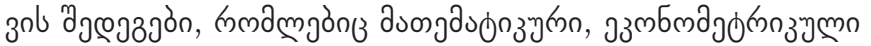

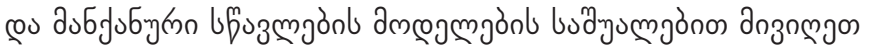

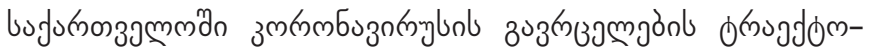

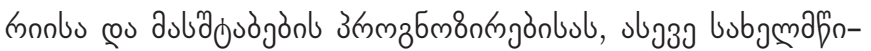

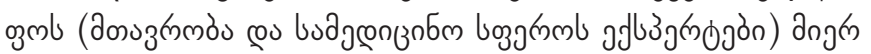

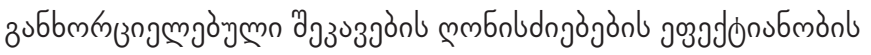

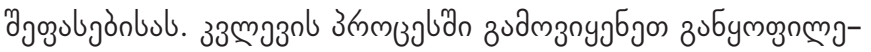

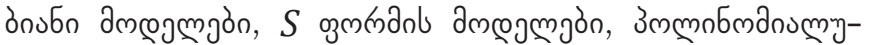

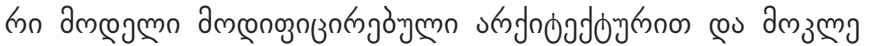

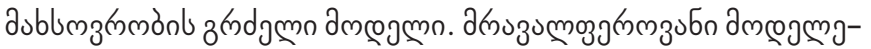

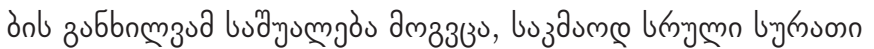
च

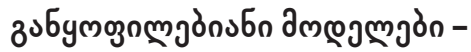

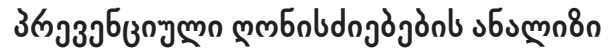

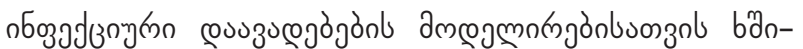

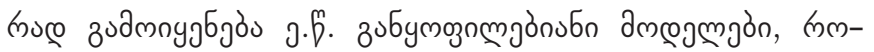

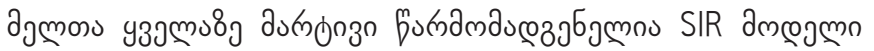
(auzumnonuce, Smith, \& Moore, 2004; Harko, T., \& al., e. 2014; Wilding, 2020; Tiwari, Kumar, \& al., e. 2020; Rahman, Ahmed, \& al., e. 2020; Chen, Lu, \& al., e. 2020; Osthus, Hickmann, K., \& al., e. 2017; Roda W., et al., 2020; Wang, Zhou, \& al., e. 2020 pou bbr.). dubzn dmbubmgmós

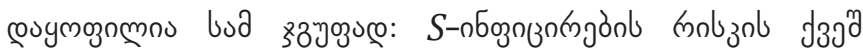
aymog

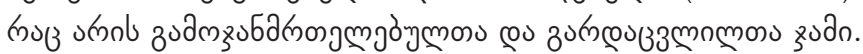

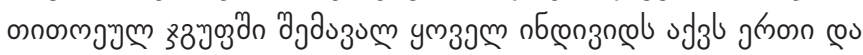

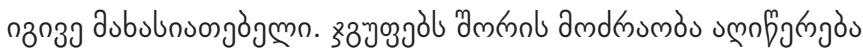

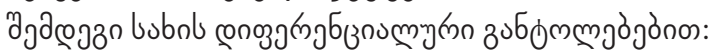

$$
\frac{d S}{d t}=-\frac{\beta S I}{N}
$$

$$
\begin{gathered}
\frac{d I}{d t}=\frac{\beta S I}{N}-\gamma I ; \\
\frac{d R}{d t}=\gamma I .
\end{gathered}
$$

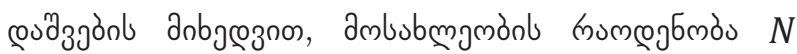

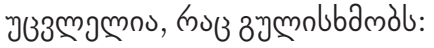

$$
\frac{d S}{d t}+\frac{d I}{d t}+\frac{d R}{d t}=0
$$

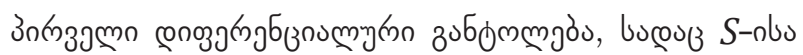

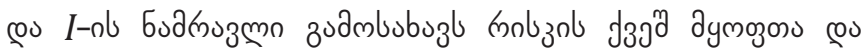

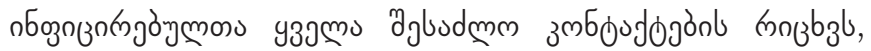

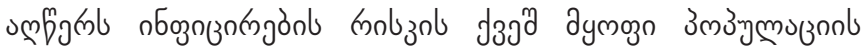

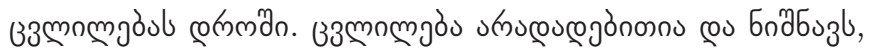

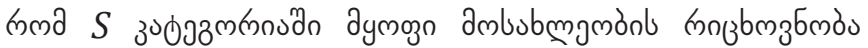

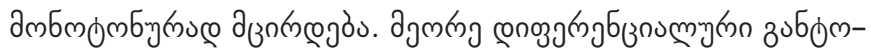

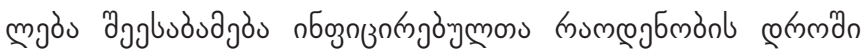

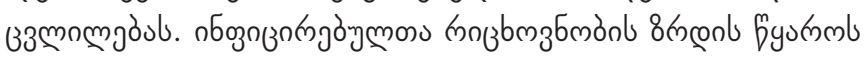

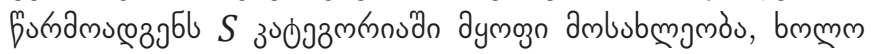

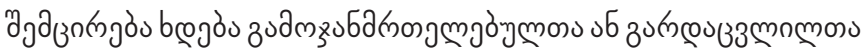

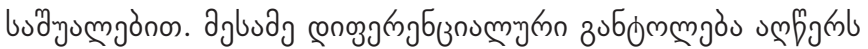

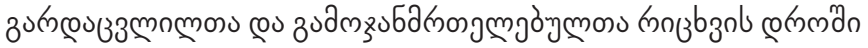

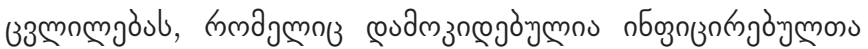

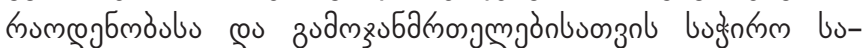

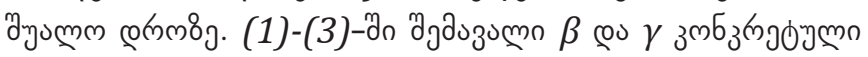

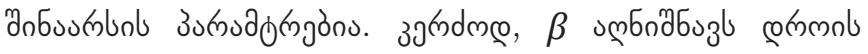

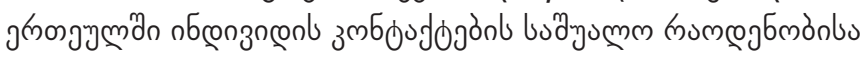

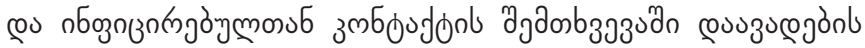

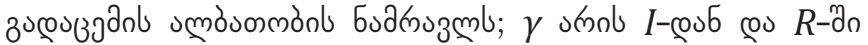

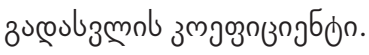

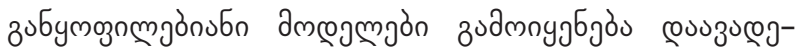

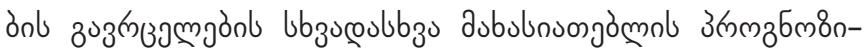

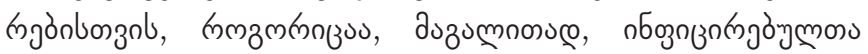

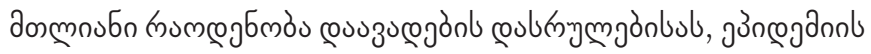

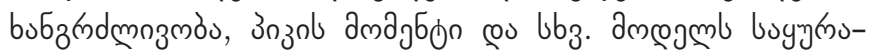

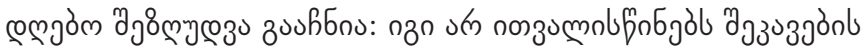

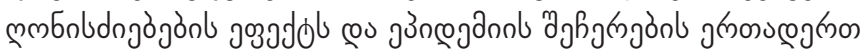

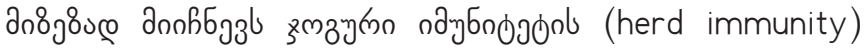

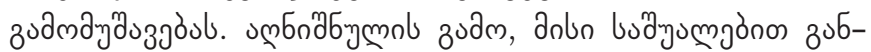

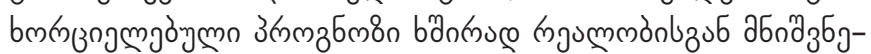

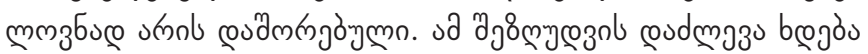

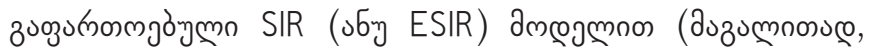
Wang, Zhou, \& al., e. 2020; Hamzah, Lau, \& al., e. 2020).

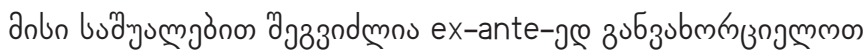

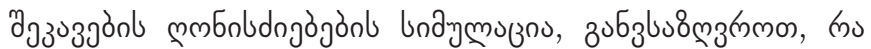

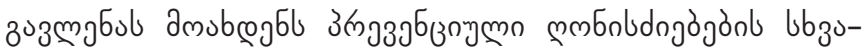

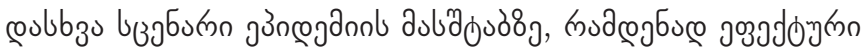

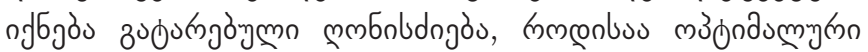

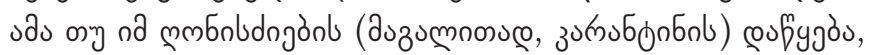




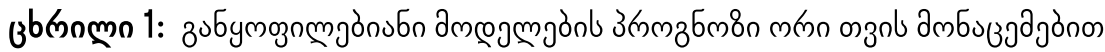

\begin{tabular}{|c|c|c|c|}
\hline & SIR & ESIR №1 & ESIR №2 \\
\hline 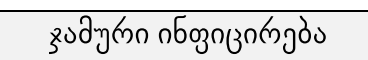 & 2854339 & 1555 & 3189 \\
\hline 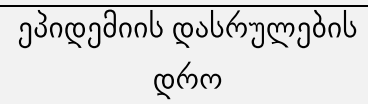 & $01 / 2021$ & 22/06/2020 & 15/07/2020 \\
\hline 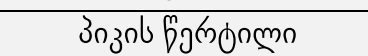 & $27 / 07 / 2020$ & $15 / 04 / 2020$ & $16 / 04 / 2020$ \\
\hline RMSE & 5976 & $\mathrm{~N} / \mathrm{A}$ & $\mathrm{N} / \mathrm{A}$ \\
\hline
\end{tabular}

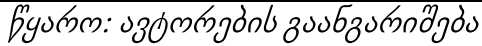

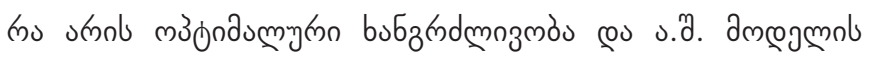

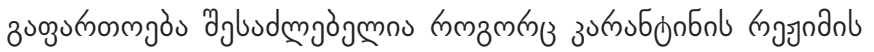

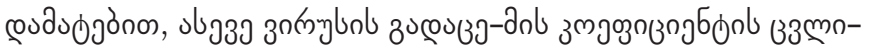

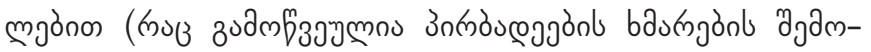

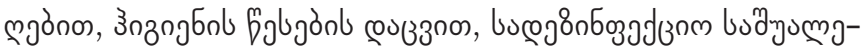

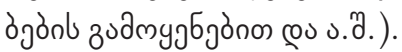

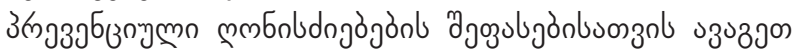

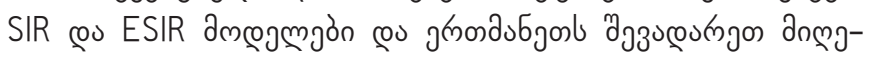

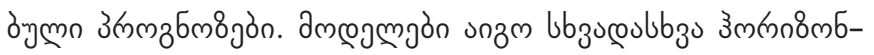

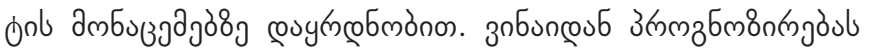
zubbuzचnor

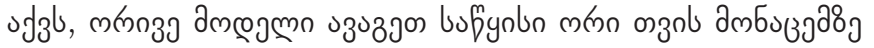

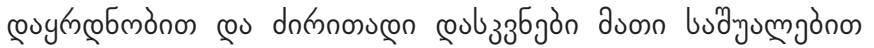

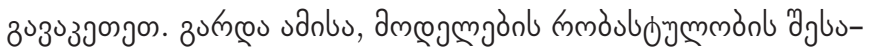

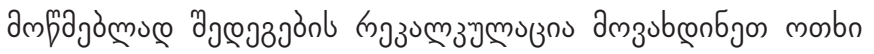

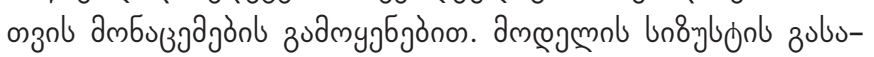

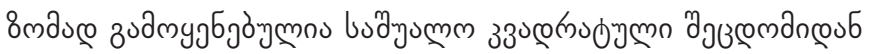
оglan

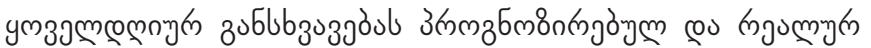

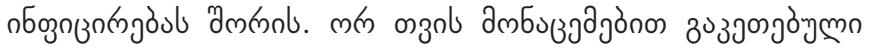

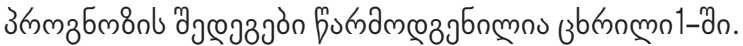

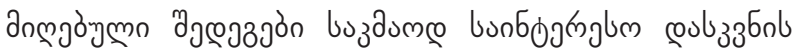

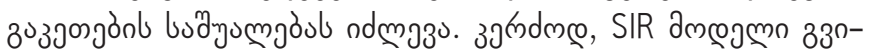

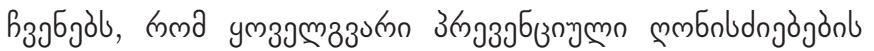

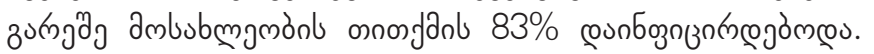

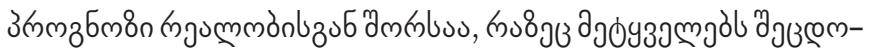

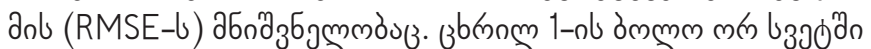

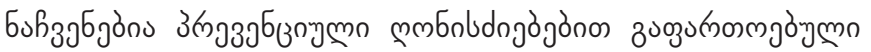

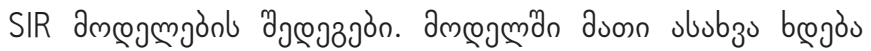

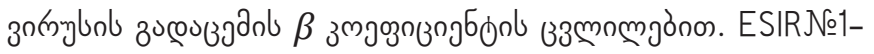

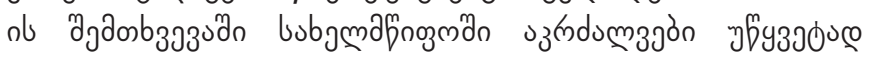

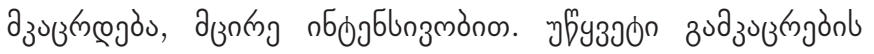

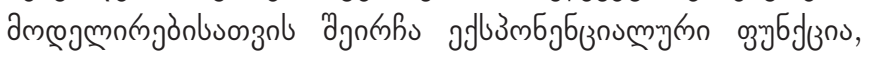

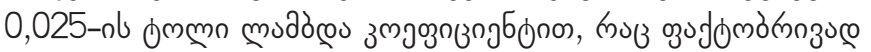

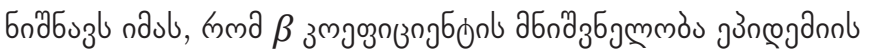

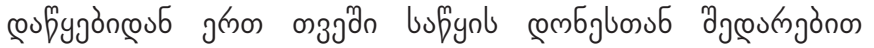

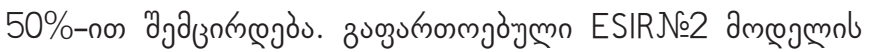

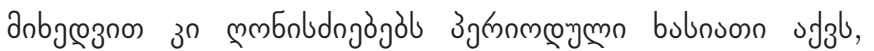

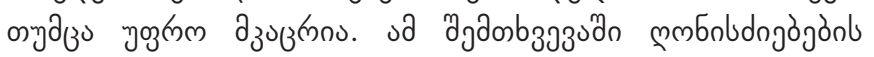

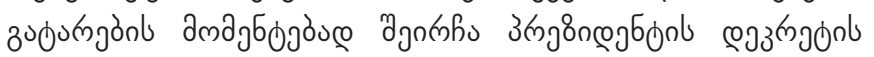

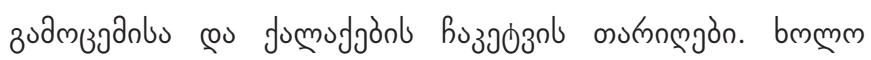

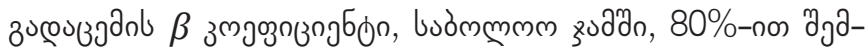

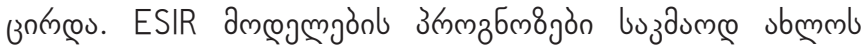

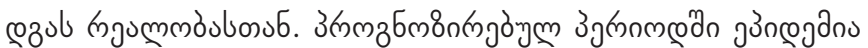

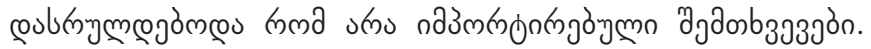

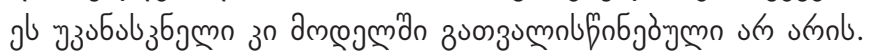

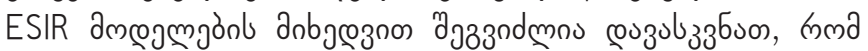

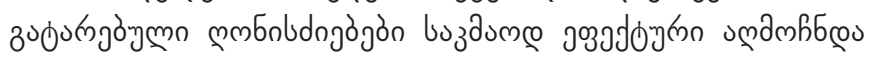

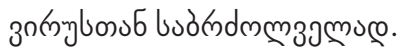

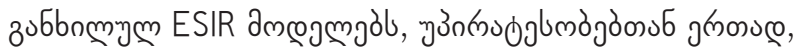

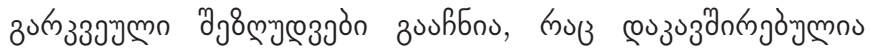

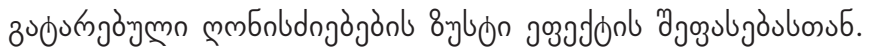

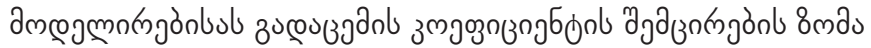

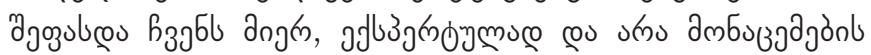

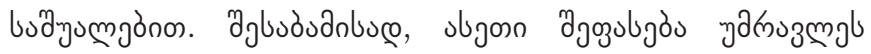

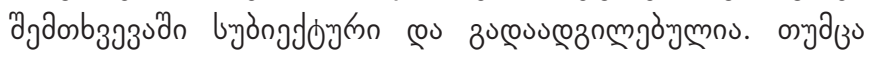

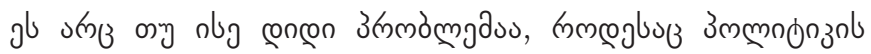

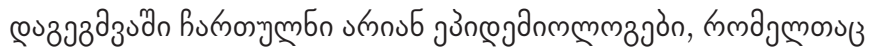

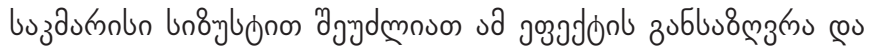

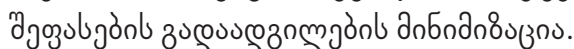

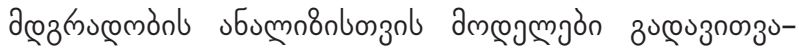

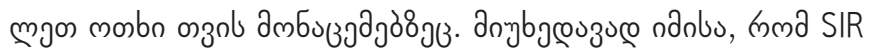

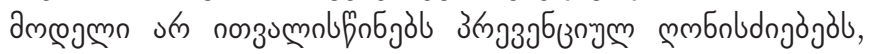

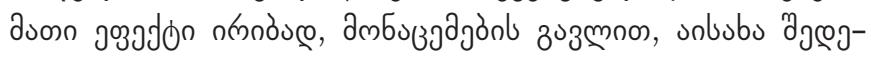

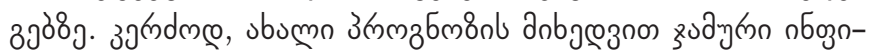

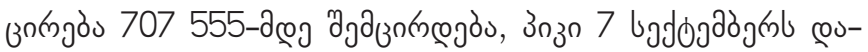

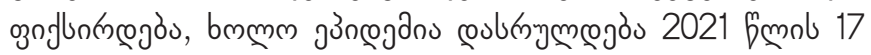

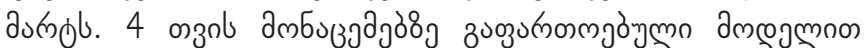

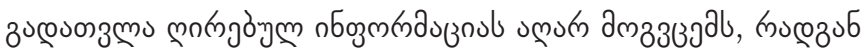

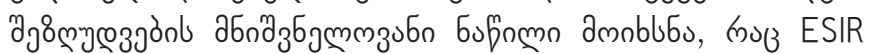
amejmb SIR amejmons denngr submmzjals (nbnmgon enszinsas 1).

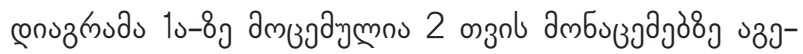

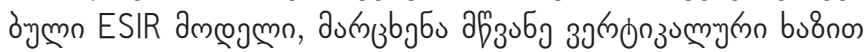

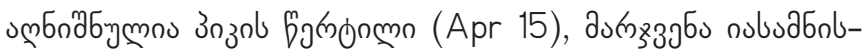

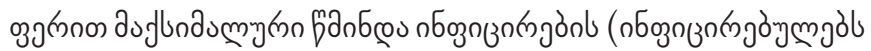

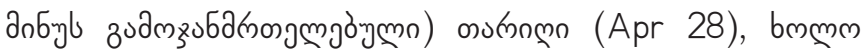

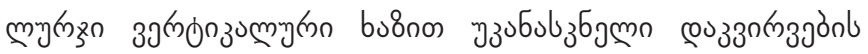

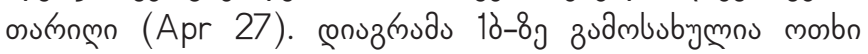

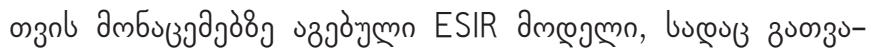




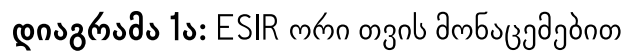

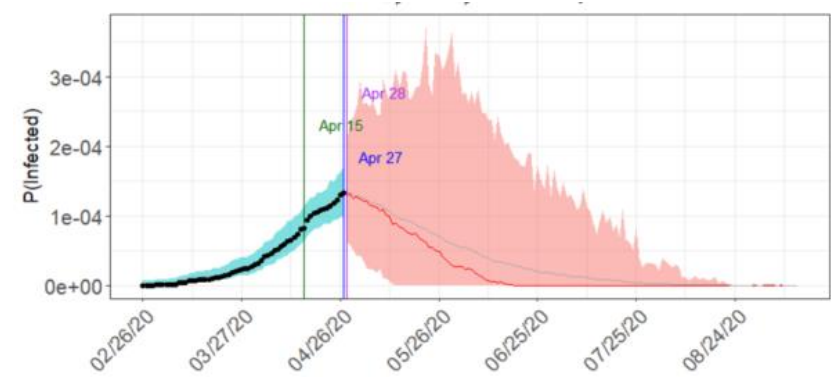

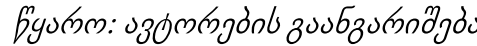

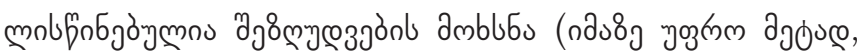

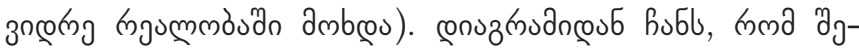

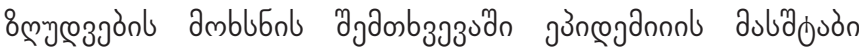

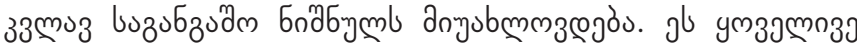

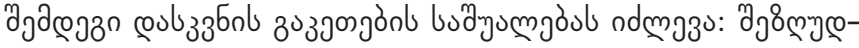

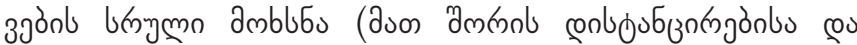

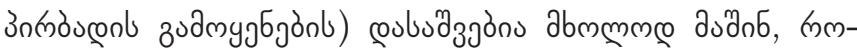

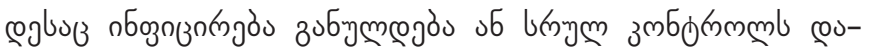

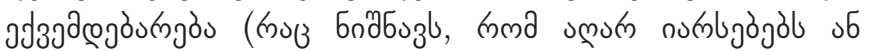

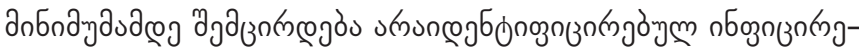

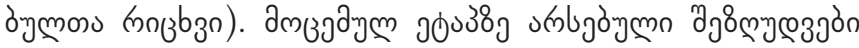

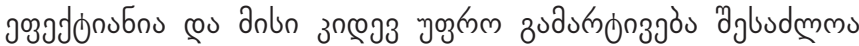

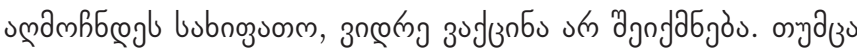

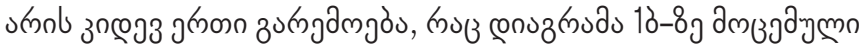

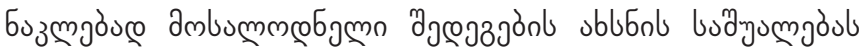

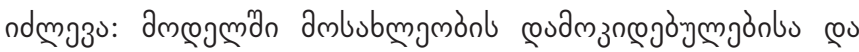

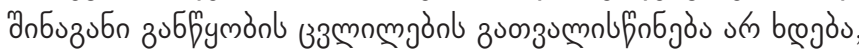

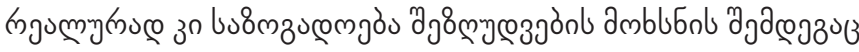

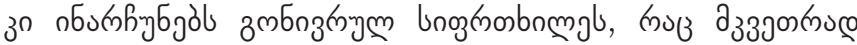

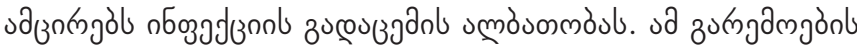

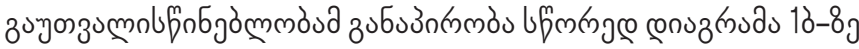

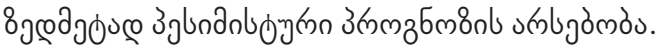

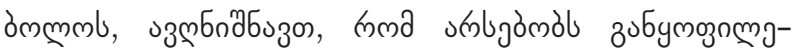

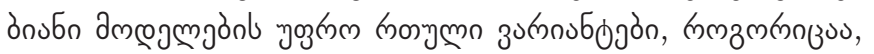

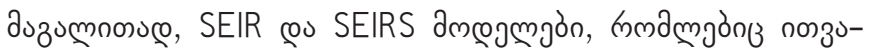

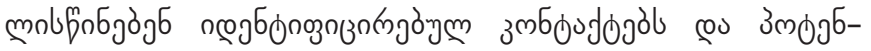

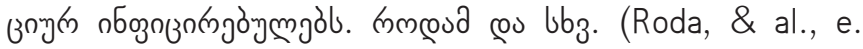

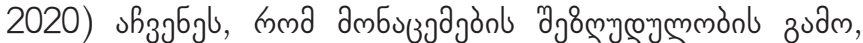

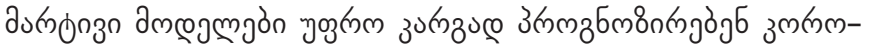

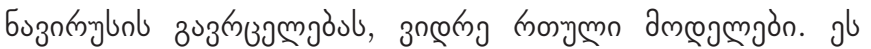

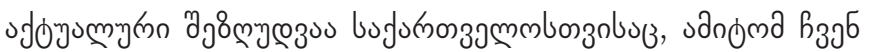

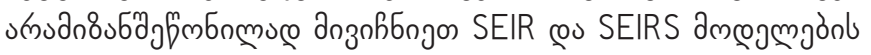

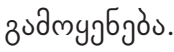

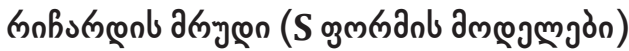

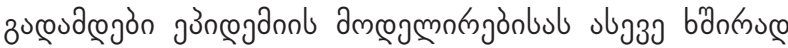

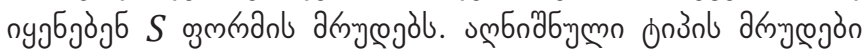

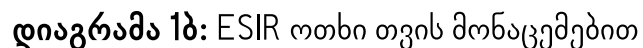

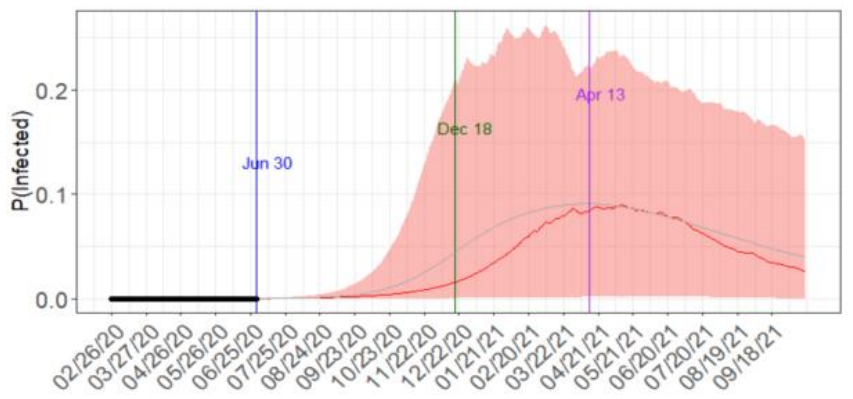

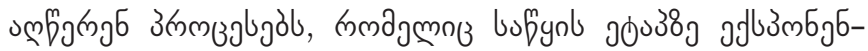

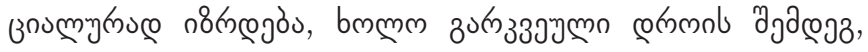

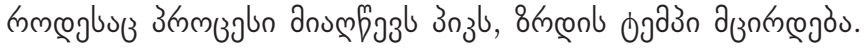

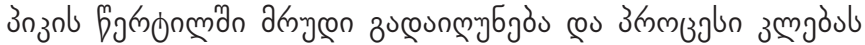
пfyjab. $S$ oुm

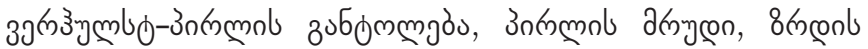

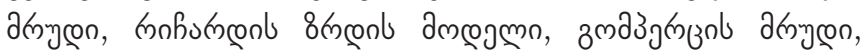

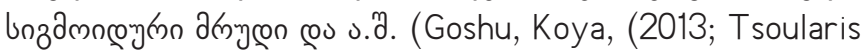
\& Wallace, 2002; Hsieh, 2009; Mazurek, \& Nenickova,

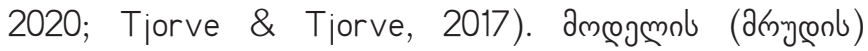

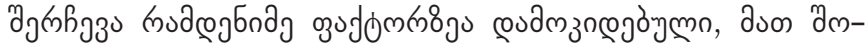

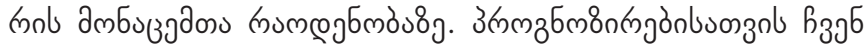

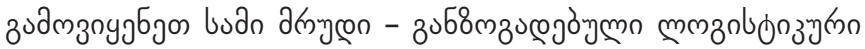

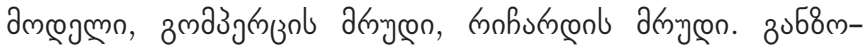

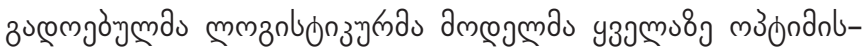
бy

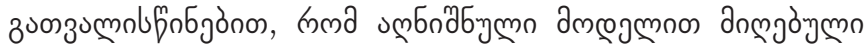

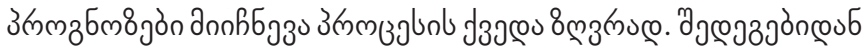

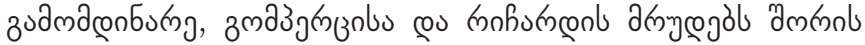
ง

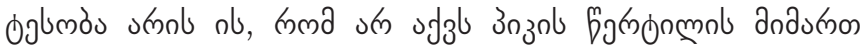

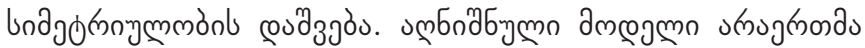

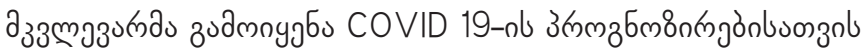
(duzumnosuc, Wu, Darcet, \& al., e. 2020; Masjedi, Rabajante, \& al., e. 2020).

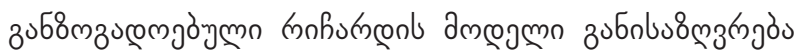

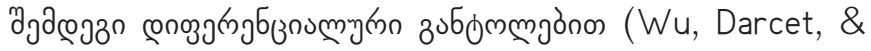
al., e. 2020):

$$
\frac{d C(t)}{d t}=r[C(t)]^{p}\left(1-\left(\frac{C(t)}{K}\right)^{a}\right),
$$

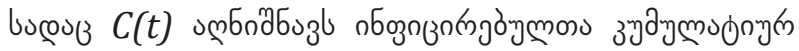

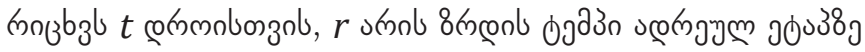

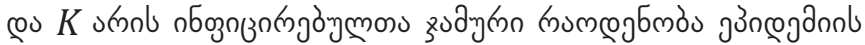

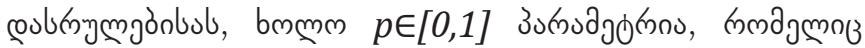

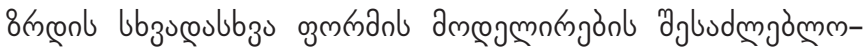




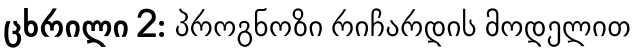

\begin{tabular}{|c|c|c|}
\hline & mohoment dmejmn №1 & 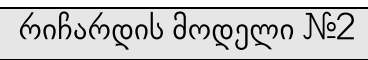 \\
\hline 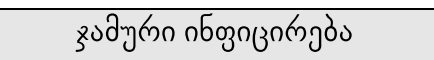 & 1317 & 1975 \\
\hline 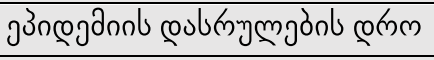 & 27 b & 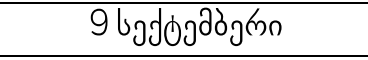 \\
\hline RMSE & 8.41 & 6.25 \\
\hline
\end{tabular}

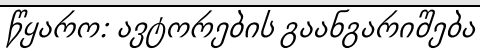

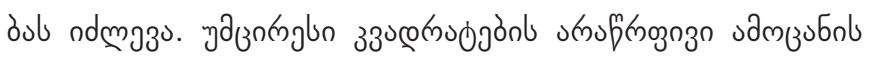

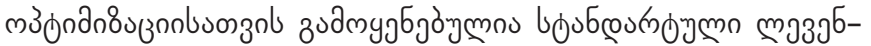

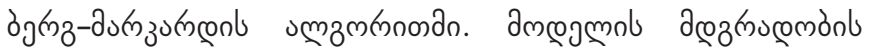

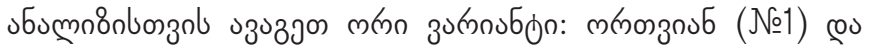

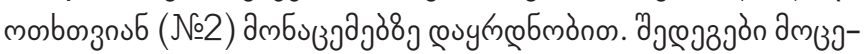

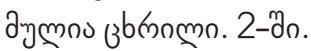

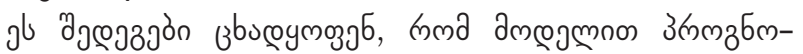

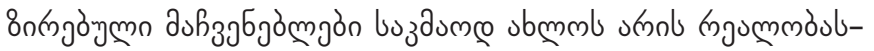

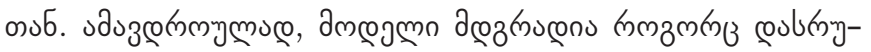

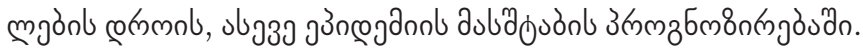

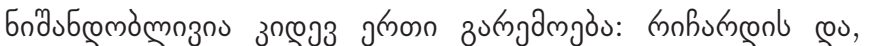
8mzu@ue, $S$ øुm

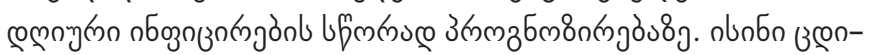
mmòj 6 3ubbu8

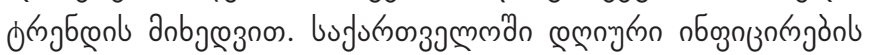

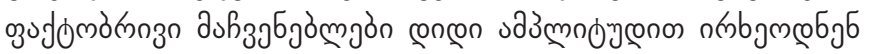

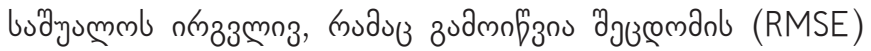

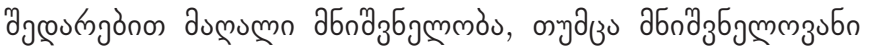

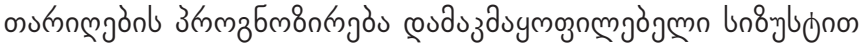

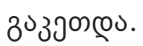

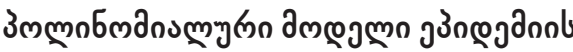

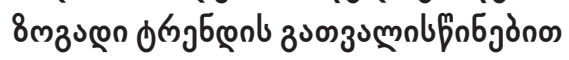

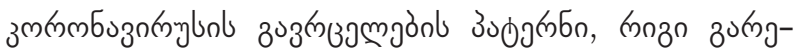

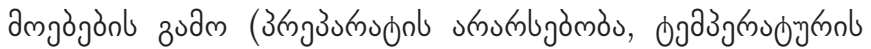

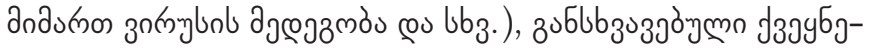

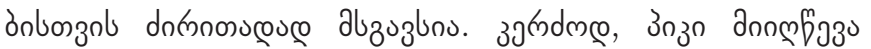

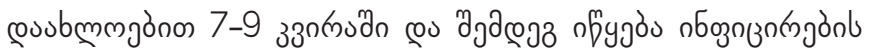

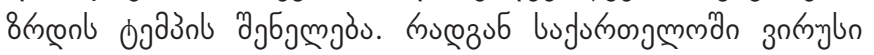

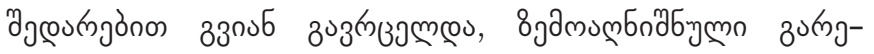

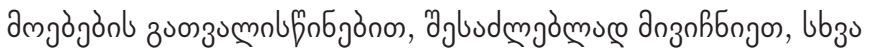

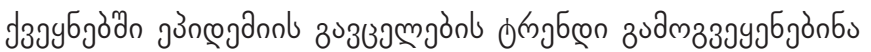
उмmzб

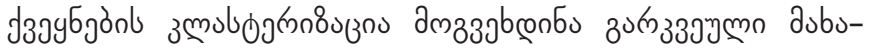

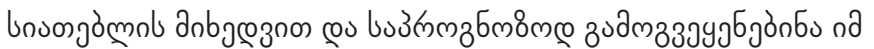

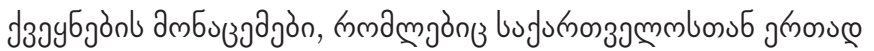

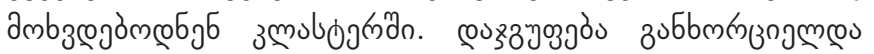

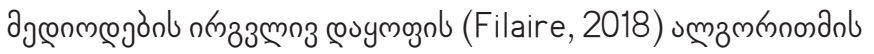

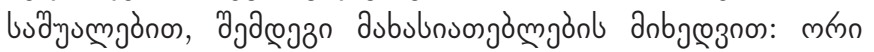

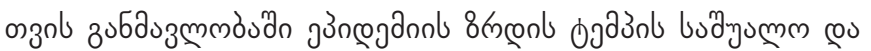

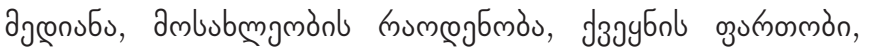

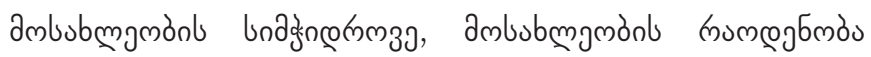

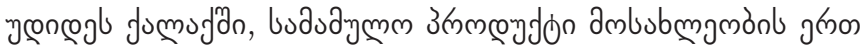

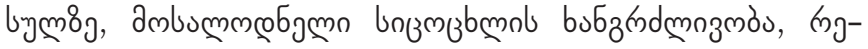

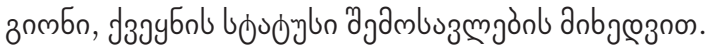

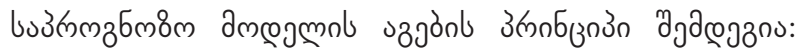

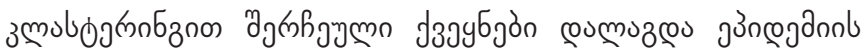

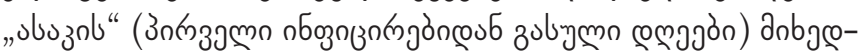

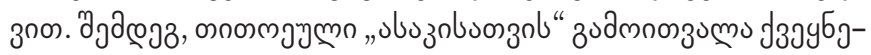

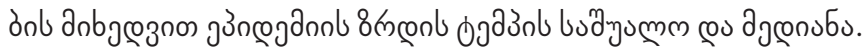

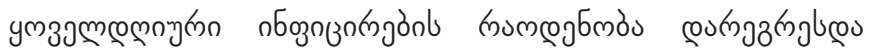

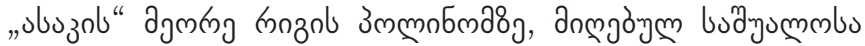

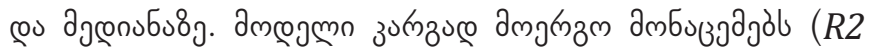

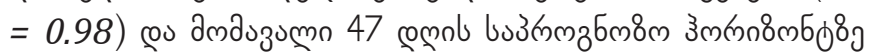

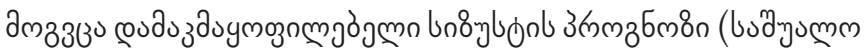

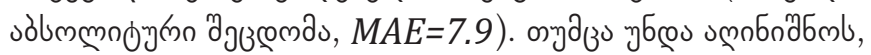

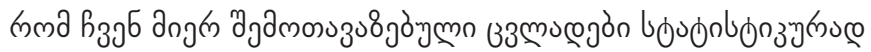

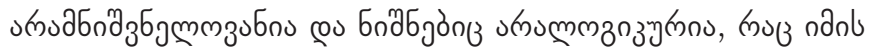

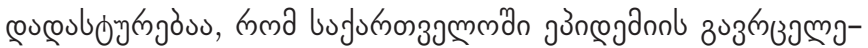

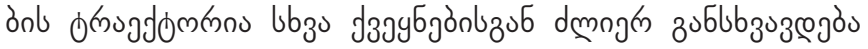

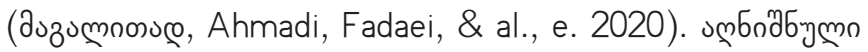

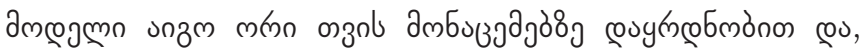

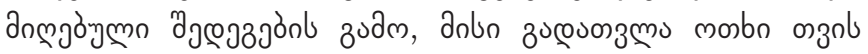

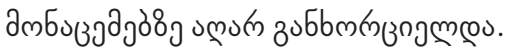

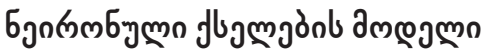

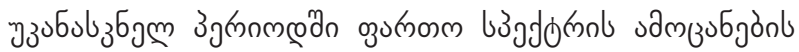

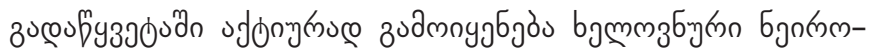

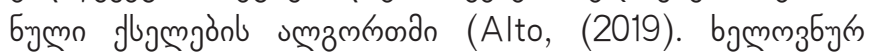

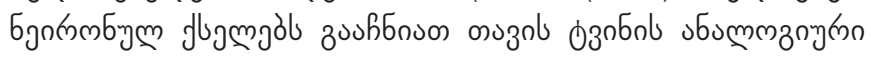

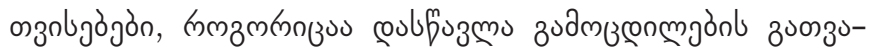

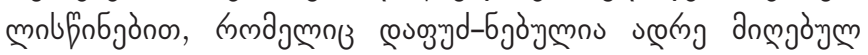

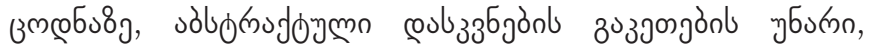

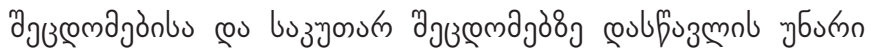

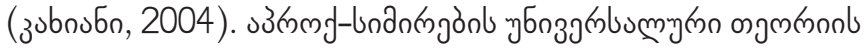

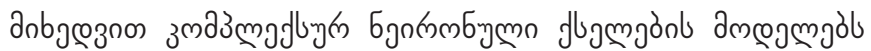

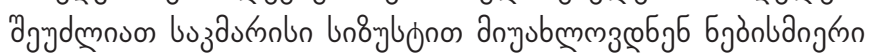

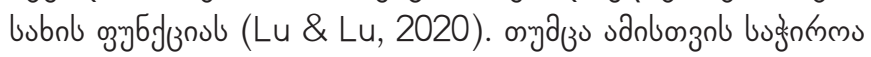
encon mumeg

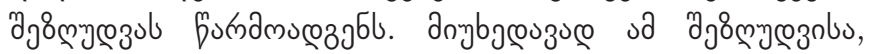

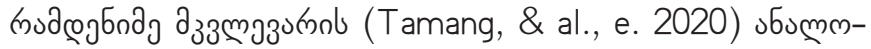

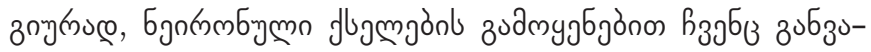




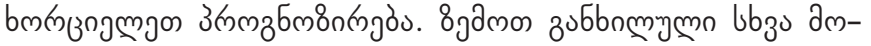

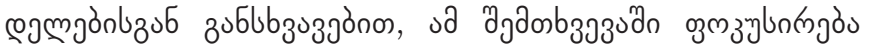

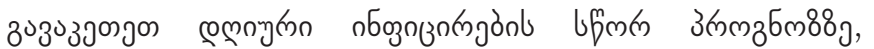

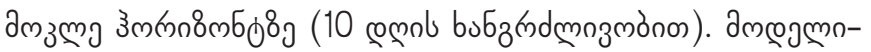

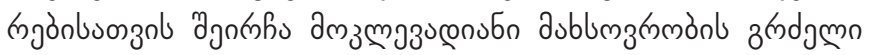

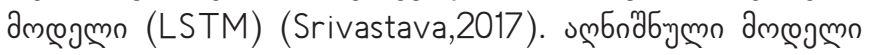

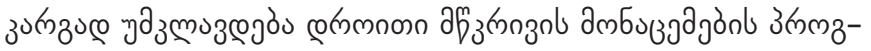

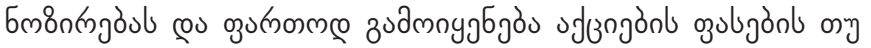

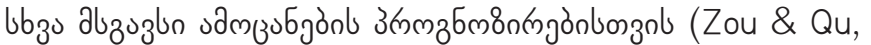

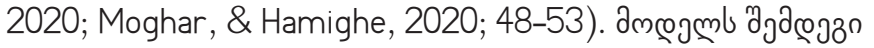

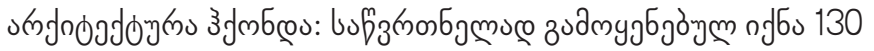

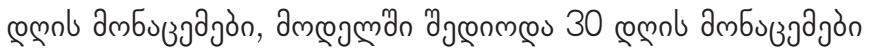

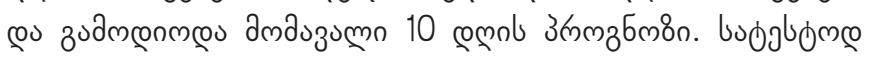

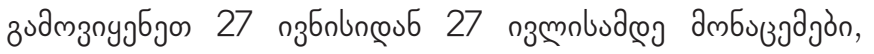

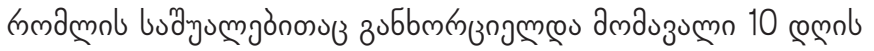

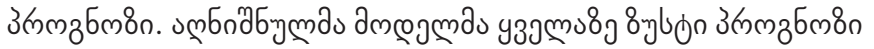

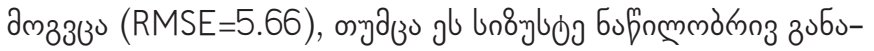

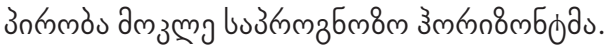

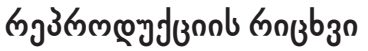

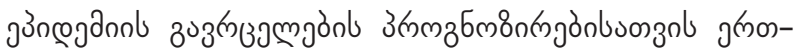

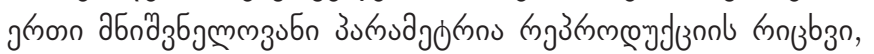

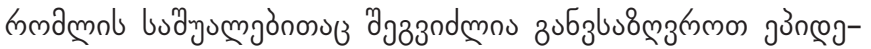

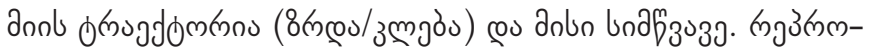

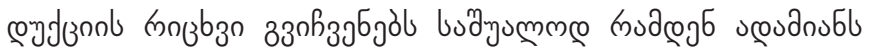

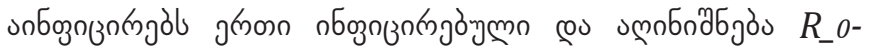

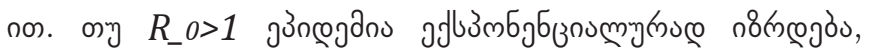

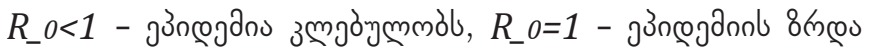

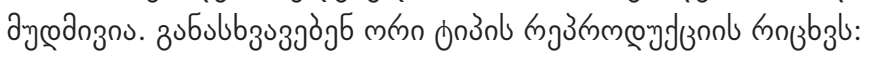

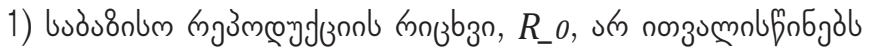

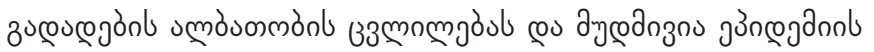

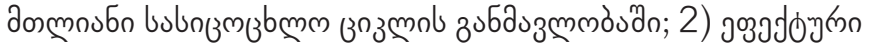

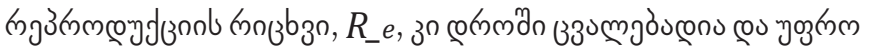

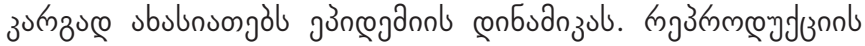

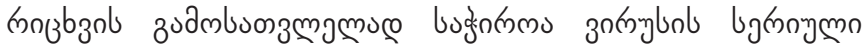

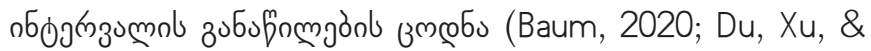

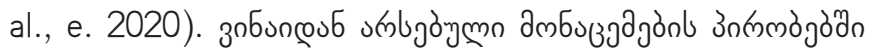

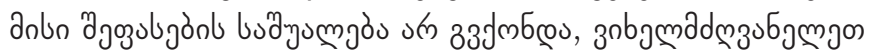

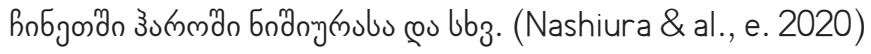

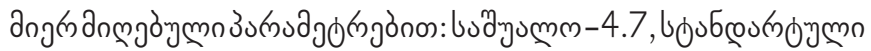

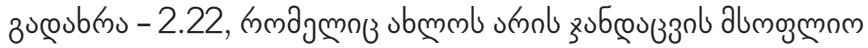

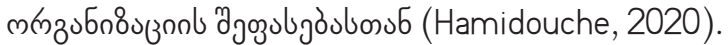

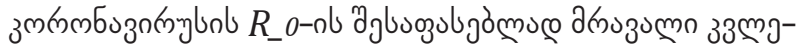

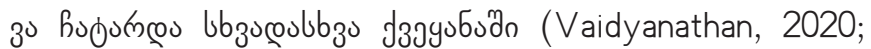
Soetewey, 2020; Ellis, 2020; Caicedo-Ochao \& al., e. 2020;

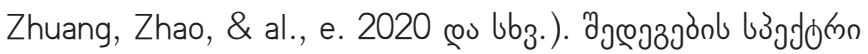

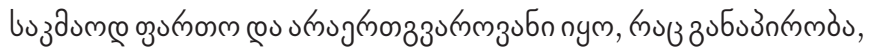

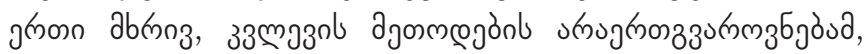
agm

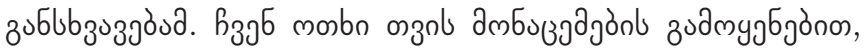

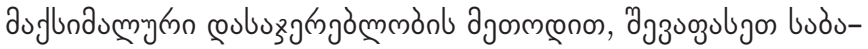

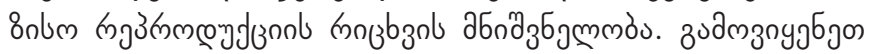
1000 gुgh

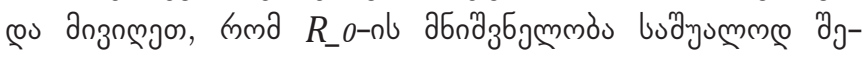

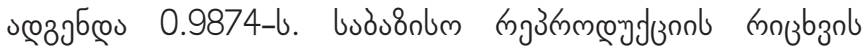

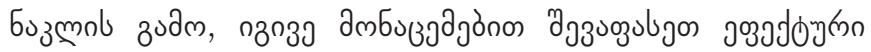

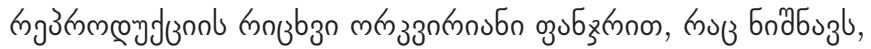

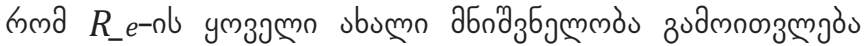

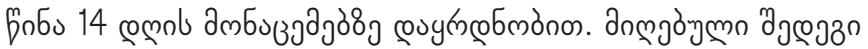

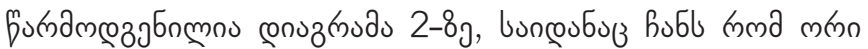

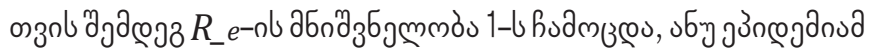
उलुjo ○ी

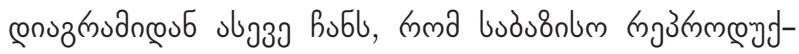

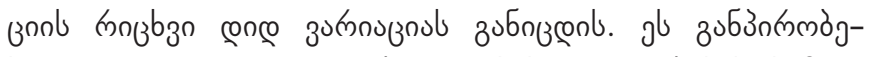

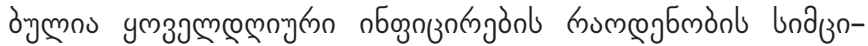

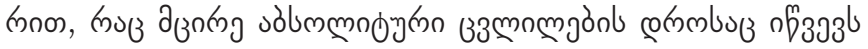

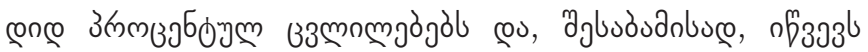

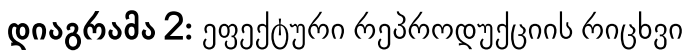

\section{Estimated R}

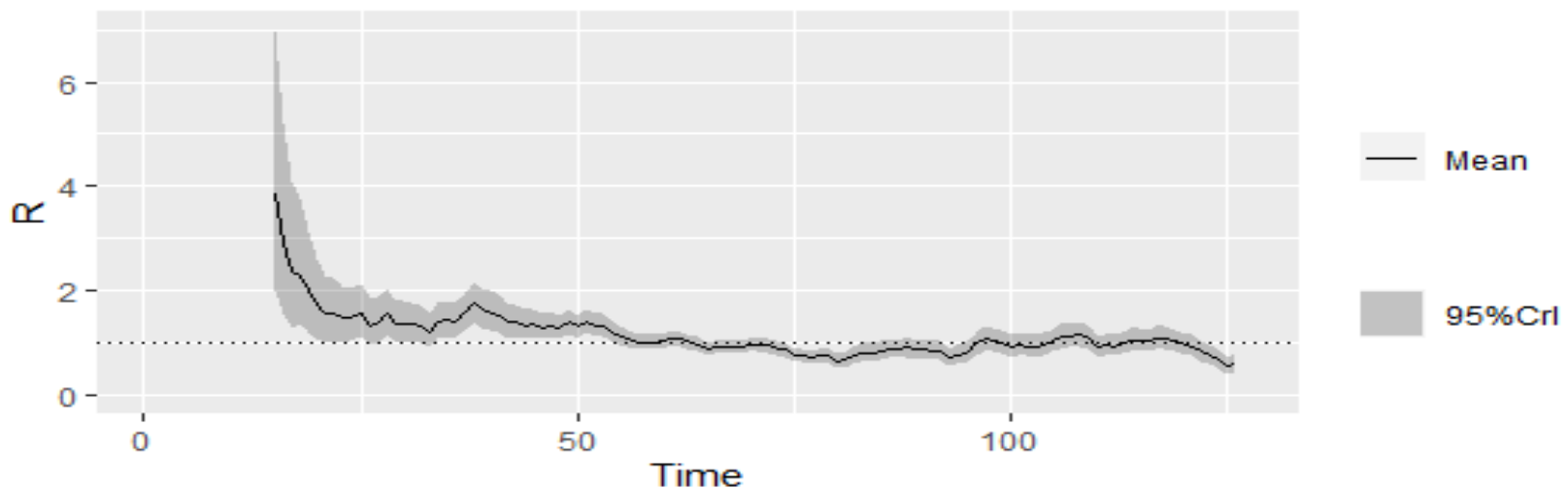

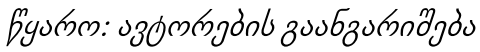




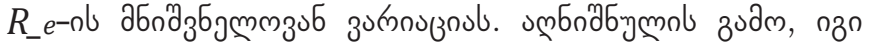

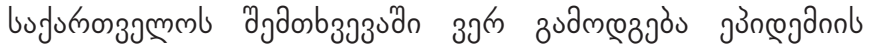

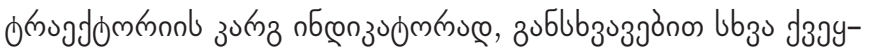

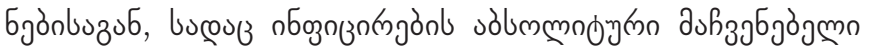
engeno.

\section{esu335s}

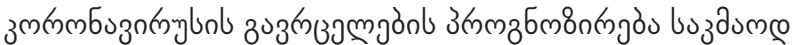

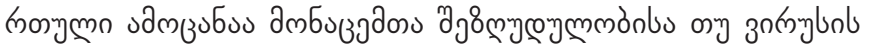

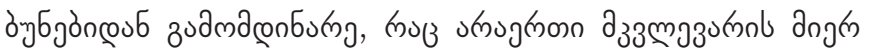

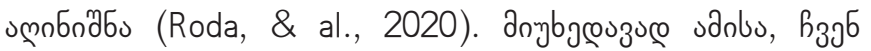

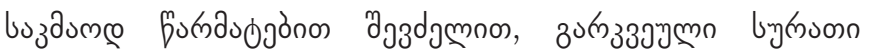

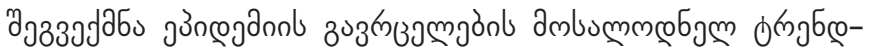

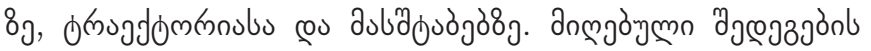

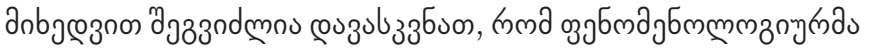

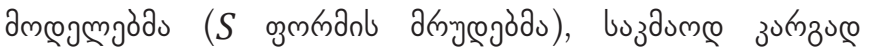

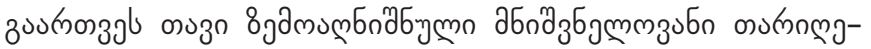

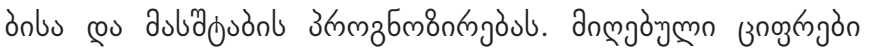

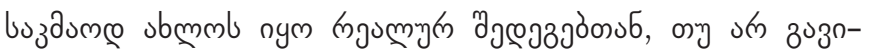

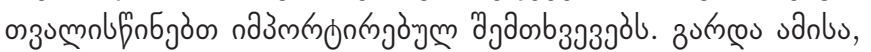

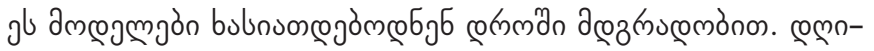

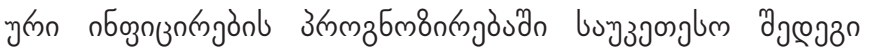

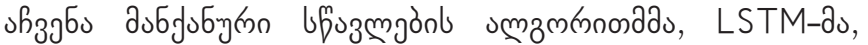

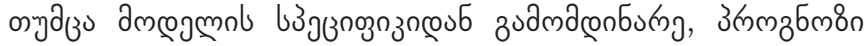

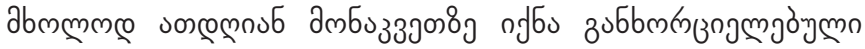

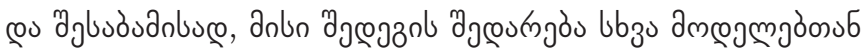

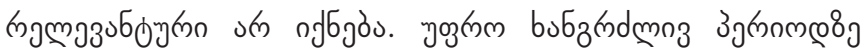

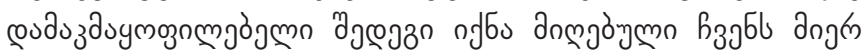

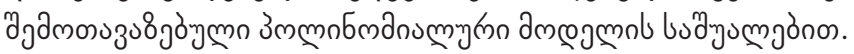

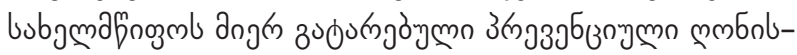

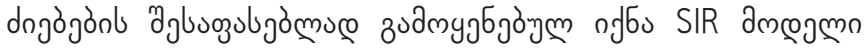

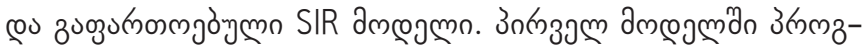

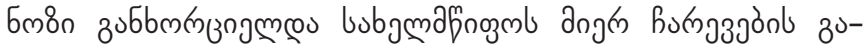

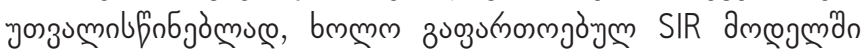

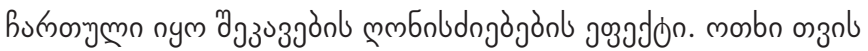

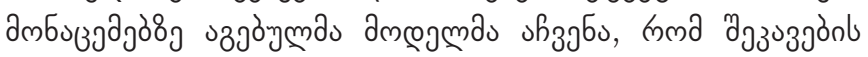

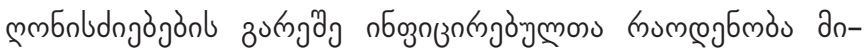

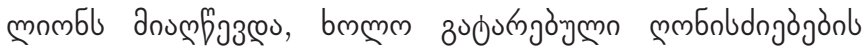

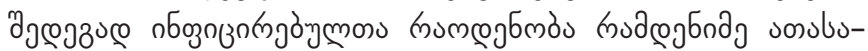

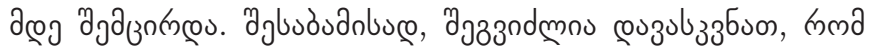

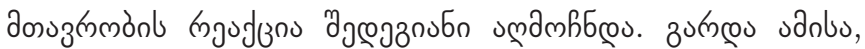

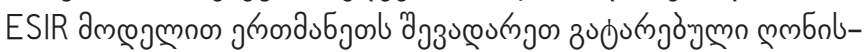

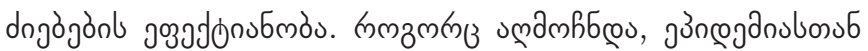

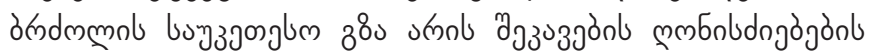

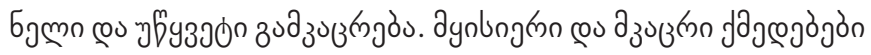

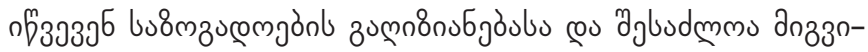

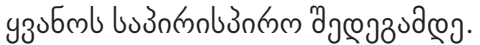

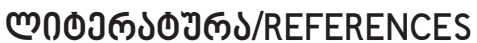

Ahmadi, A., Fadaei, Y., \& al., e. (2020). Modeling and Forecasting Trend of COVID-19 Epidemic in Iran until May 13, 2020. Medical Journal of the Islamic Republic of Iran. 2020, 34:27. Available at: https://www.ncbi.nlm.nih.gov/pmc/articles/ PMC7320984/

Alto, V. (2019). Neural Networks: parameters, hyperparameters and optimization strategies. Available at: https:// towardsdatascience.com/neural-networks-parameters-hyperparameters-and-optimization-strategies-3f0842fac0a5

Baum, S. (2020). Serial Interval of COVID-19. Available at: https://www.jwatch.org/na51171/2020/03/27/serial-intervalcovid-19

Caicedo-Ochao, Y., \& al., e. (2020). Effective Reproductive Number estimation for initial stage of COVID-19 pandemic in Latin American Count. International Journal of Infectious Diseases 95 (2020). 316-318.

Chen, Y.-C., Lu, P.-E., \& al., e. (2020). A Time-dependent SIR model for COVID-19 with Undetectable Infected Persons. Available at: https://www.researchgate.net/publication/339642417_

Du, Z., Xu, X., \& al., e. (2020). serial Interval of COVID-19 among Publicly Reported Confirmed Cases. Emerg Infect Dis. 2020;26(6):1341-1343. doi:10.3201/eid2606.200357

Ellis, P. (2020). Test positivity rates and actual incidence and growth of diseases. Available at: http://freerangestats.info/ blog/2020/05/09/covid-population-incidence.

Filaire, T. (2018). Clustering on mixed type data. Available at: https://towardsdatascience.com/clustering-on-mixed-typedata-8bbd0a2569c3

Goshu, A.T. and Koya, P.R. (2013) Derivation of Inflection Points of Nonlinear Regression Curves-Implications to Statistics. American Journal of Theoretical and Applied Statistics, 2, 268-272. Available at: http://dx.doi.org/10.11648/j. ajtas. 20130206.25

Hamidouche, M. (2020). COVID-19 outbreak in Algeria: A mathematical Model to predict cumulative cases. Available at: https://www.medrxiv.org/content/10.1101/2020.03.20.20039891v2

Hamzah, F. A., Lau, C. H., \& al., e. (2020). CoronaTracker: World-wide COVID-19 Outbreak Data Analysis and Prediction. Available at: https://www.who.int/bulletin/online_first/20-255695.pdf

Harko, T., \& al., e. (2014). Exact analytical solutions of the Susceptible-Infected-Recovered (SIR) epidemic model and of the SIR 
model with equal death and birth rates. Applied Mathematics and Computation 236(2014):184-194.

Hsieh, Y.-H. (2009). Richards Model: A Simple Procedure for Real-time Prediction of Outbreak Severity. In Modeling and dynamics of infectious diseases, pages 216-236. World Scientific.

Kakhiani, G. (2004). Creating adaptive forecasting algorithms using artificial neural networks. (Prognozirebis adapturi algoritmebis sheqmna khelovnuri qselebis gamoyenebit). (In Georgian). Available at: https://www.google.com/ search?source=univ\&tbm=isch\&q=

Lu, J., \& Lu, Y. (2020). A Universal Approximation Theorem of Deep Neural Networks for Expressing Distributions. Available at: https://arxiv.org/pdf/2004.08867.pdf

Masjedi, H., Rabajante, J., \& al., e. (2020). Nowcasting and Forecasting the Spread of COVID-19 in Iran. Available at: file:///C:/ Users/PC-USER/Downloads/Nowcasting_and_Forecasting_the_Spread_of_COVID-19_.pdf

Mazurek, J., \& Nenickova, Z. (2020). Predicting the number of total COVID-19 cases and deaths in the USA by the Gompertz curve. Available at: https://www.researchgate.net/publication/341132093

Moghar, A., \& Hamiche, M. (2020). Stock Market Prediction Using LSTM Recurrent Neural Network. Available at: https:// www.researchgate.net/publication/340636297_

Nashiura, H., \& al., e. (2020). Serial interval of novel coronavirus (COVID-19) infections. International Journal of Infectious Diseases 93(2020) 284-286.

Osthus, D., Hickmann, K., \& al., e. (2017). Forecasting seasonal influenza with a state-space SIR model. Available at: https:// projecteuclid.org/download/pdfview_1/euclid.aoas/1491616878

Rahman, M., Ahmed, A., \& al., e. (2020). Impact of control strategies on COVID-19 pandemic and the SIR model based forecasting in Bangladesh. Available at: https://www.researchgate.net/publication/340879877_

Roda W., et al., (2020). Why is it difficult to accurately predict the COVID-19 epidemic? Infectious Disease Modelling 5 (2020), $271-281$

Round Table "Coronomics", (2020). (In Georgian). Economics and Business, (2020), 2. Available at: http://eb.tsu. ge/?leng=ge\&cat=nom

Smith, D., \& Moore, L. (2004). The SIR Model for Spread of Disease - The Differential Equation Model. Available at: https:// www.maa.org/press/periodicals/loci/joma/the-sir-model-for-spread-of-disease-the-differential-equation-model

Soetewey, A. (2020, March). COVID-19 in Belgium. Available at: https://www.statsandr.com/blog/covid-19-in-belgium/

Srivastava, P. (2017). Essentials of Deep Learning : Introduction to Long Short Term Memory. Available at: https://www. analyticsvidhya.com/blog/2017/12/fundamentals-of-deep-learning-introduction-to-Istm/

Tamang, M., \& al., e. (2020). A Machine-Learning-Based Approach to Predict the Health Impacts of Commuting in Large Cities: Case Study of London, Symmetry 2020, 12, 866; doi:10.3390/sym12050866

Tiwari, S., Kumar, S., \& al., e. (2020). Outbreak Trends of Coronavirus Disease-2019 in India: A predicion. Disaster Med Public Health Prep. 2020 Apr 22: 1-6. Published online 2020 Apr 22. doi: 10.1017/dmp.2020.115. Available at: https://www. ncbi.nlm.nih.gov/pmc/articles/PMC7211803/

Tjorve K., Tjorve E. (2017) The use of Gompertz models in growth analyses, and new Gompertz-model approach: An addition to the Unified-Richards family. PLOS ONE 12(6): e0178691. Available at: https://doi.org/10.1371/journal.pone.

Tsoularis, A., \& Wallace, J. (2002). Analysis of Logistic Growth Models. Matematical Biosciences, 179, 1. July-August, 21-55

Vaidyanathan, R. (2020). Estimating COVID-19's Rt in Real-Time (Replicating in R).Available at: https://www.datacamp.com/ community/tutorials/replicating-in-r-covid19

Wang, L., Zhou, Y., \& al., e. (2020). An epidemiological forecast model and software assessing interventions on COVID-19

epidemic in China. Available at: https://www.medrxiv.org/content/10.1101/2020.02.29.20029421v1.full.pdf+html Wilding,

T. (2020). Epidemic modelling of COVID-19 in the UK using a SIR model. Available at: https://tjwilding.wordpress. $\mathrm{com} / 2020 / 03 / 20 /$

Wu, K., Darcet, D., \& al., e. (2020). Generalized logistic growth modeling of the COVID-19 outbreak in 29 provinces in China and in the rest of the world. Available at:

https://www.medrxiv.org/content/10.1101/2020.03.11.20034363v1

Zhuang, Z., Zhao, S., \& al., e. (2020). Preliminary estimates of the reproduction number of the coronavirus disease (COVID-19) outbreak in Republic of Korea and Italy by 5 March 2020. International Journal of Infectious Diseases. 95, (2020), 308310.

Zou, Z., \& Qu, Z. (2020, January). Using LSTM in Stock prediction and Quantitative Trading. Available at: http://cs230.stanford. edu/projects_winter_2020/reports/32066186.pdf

https://www.kaggle.com/pablocastilla/predict-stock-prices-with-lstm

https://analyticsindiamag.com/hands-on-guide-to-Istm-recurrent-neural-network-for-stock-market-prediction/

https://medium.com/towards-artificial-intelligence/google-stock-predictions-using-an-lstm-neural-network-dbe785949a96

https://github.com/CSSEGISandData/COVID-19/tree/master/csse_covid_19_data/csse_covid_19_time_series 